\title{
La Résilience urbaine : un nouveau concept opérationnel vecteur de durabilité urbaine?
}

Urban resilience: is it a useful concept for urban sustainability?

Marie Toubin, Serge Lhomme, Youssef Diab, Damien Serre et Richard Laganier

\section{(2) OpenEdition}

12 Journals

Édition électronique

URL : http://journals.openedition.org/developpementdurable/9208

DOI : 10.4000/developpementdurable.9208

ISSN : 1772-9971

Éditeur

Association DD\&T

Référence électronique

Marie Toubin, Serge Lhomme, Youssef Diab, Damien Serre et Richard Laganier, « La Résilience urbaine : un nouveau concept opérationnel vecteur de durabilité urbaine ?», Développement durable et territoires [En ligne], Vol. 3, n 1 | Mai 2012, mis en ligne le 24 mai 2012, consulté le 10 décembre 2020. URL : http://journals.openedition.org/developpementdurable/9208 ; DOI : https://doi.org/10.4000/ developpementdurable.9208

Ce document a été généré automatiquement le 10 décembre 2020.

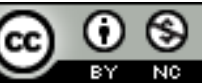

Développement Durable et Territoires est mis à disposition selon les termes de la licence Creative Commons Attribution - Pas d'Utilisation Commerciale 4.0 International. 


\title{
La Résilience urbaine : un nouveau concept opérationnel vecteur de durabilité urbaine?
}

\author{
Urban resilience : is it a useful concept for urban sustainability?
}

Marie Toubin, Serge Lhomme, Youssef Diab, Damien Serre et Richard Laganier

1 L'année 2011 atteint déjà des records en matière de coûts liés aux catastrophes naturelles (Catnat.net, 2011). Chaque année, les rapports des réassureurs alertent quant à l'augmentation des montants indemnisés et les médias passent d'une catastrophe à une autre, présentant des images chocs, des témoignages bouleversants, sans jamais se pencher plus précisément sur les causes profondes, les conséquences à long terme, la reconstruction et les conditions de retour à la vie normale pour les populations touchées. Or si les dommages augmentent, c'est notamment parce que les enjeux (logements, activités, infrastructures, équipements) sont de plus en plus nombreux dans les zones d'aléas, comme l'atteste entre autres la forte urbanisation des zones inondables (Faytre, 2010; Scarwell et Laganier, 2004; Thouret et Leone, 2003). L'intensification des évènements climatiques extrêmes semble également à l'œuvre, avec une responsabilité probable du changement climatique, même si celle-ci reste difficilement quantifiable. Toujours est-il que les populations, et en particulier les populations urbaines, vivent mal les crises lorsqu'elles surviennent. Si les niveaux de risque augmentent, le degré d'acceptabilité sociale du risque reste faible au point que la directive européenne inondation demande aux Etats membres de considérer désormais une crue centennale non comme un évènement extrême mais plutôt comme un événement de niveau moyen. Les niveaux de protection à assurer sont donc de plus en plus coûteux et impactent profondément le fonctionnement de la ville. Ne voit-on pas dès lors apparaître une certaine contradiction entre des politiques de gestion des risques qu'il conviendrait d'adapter et un développement urbain qui prend rarement en compte les potentielles défaillances en cas de crise ? Deux concepts ont émergé successivement pour caractériser la ville et interroger ses contradictions, l'un prenant en compte la gestion des 
perturbations : la ville résiliente, l'autre prônant un développement économique, social et environnemental équilibré : la ville durable. A partir de leur définition et surtout de l'acception opérationnelle que nous en faisons, cet article interroge l'articulation des deux concepts à travers un point de vue technique et fonctionnel. Ce positionnement n'est pas fondamentalement issu de l'interprétation du concept de résilience mais plutôt de l'appréhension de l'objet d'étude auquel il est appliqué, à savoir la ville. Ces réflexions théoriques permettent ensuite d'évoquer les pistes méthodologiques pour concrétiser la ville durable par une approche in fine socio-technique de l'adaptation du système urbain et de ses composants (notamment les réseaux techniques).

\section{Ville résiliente ou ville durable?}

2 L'émergence de ces deux notions floues mais pourtant souvent invoquées nécessite de clarifier leurs acceptions et leur possible articulation. Les acceptions originelles de la notion de durabilité et les diverses définitions de la résilience qui amènent à choisir ici une approche relativement technique pour en garantir l'opérationnalité, feraient de la première un idéal urbain à atteindre, de la seconde, un outil, un moyen de concrétiser cet idéal car « les aspirations non-focalisées de la durabilité sont incluses dans la notion de résilience - la capacité de persister et de s'adapter $»^{1}$ (Adger, 2003).

\subsection{La durabilité urbaine}

3 Le développement durable, dans sa définition première, a pour objectif de ne pas compromettre le développement des générations futures tout en corrigeant les formes actuelles d'inégalités de développement entre les territoires. Le développement durable serait alors l'articulation d'un principe objectif d'interdépendance et d'un principe normatif d'équité spatiale et temporelle (Laganier et al., 2002). Le concept est donc hautement anthropocentriste et pour partie subjectif. La recherche de la durabilité induit en effet un jugement de valeur, moral, quant aux objectifs désirables, aux territoires concernés, à l'échelle de temps choisie. En outre, la dialectique entre les notions de durabilité et de perturbation n'est pas évidente compte-tenu des échelles temporelles auxquelles elles font références (temps long et temps court) et des valeurs qu'elles mobilisent. Même si l'émergence de la notion de durabilité coïncide avec l'émergence de la « société du risque », aujourd'hui le volet " gestion des risques » bien que transversal et s'inscrivant dans la durée, est loin d'occuper une place centrale dans le développement durable (Casteigts, 2008). En effet, si la durabilité urbaine ne peut pas s'affranchir des questions de perturbation ou d'instabilité, elles ne constituent pas le fondement de sa construction et la notion intègre plutôt des questions d'incertitudes concernant les besoins futurs ou l'évolution du contexte environnemental. Dès lors, un développement peut-il être durable en période de crise, lorsque la priorité est donnée à la protection des personnes et des biens, parfois au détriment de l'économie ou de l'environnement ? Doiton même rechercher le développement (de quoi ?) dans ces situations d'incertitude et d'urgence?

Face à ces interrogations, comment aborder la durabilité de la ville ? Le développement durable des sociétés humaines, issu des travaux du sommet de Rio en 1992, interroge aujourd'hui la ville dans ses différentes dimensions matérielles, fonctionnelles, sociales, économiques, politiques. Dès lors, une première contradiction apparaît : la ville ne peut 
pas être durable dans ses limites administratives (Mori et Christodoulou, 2011). Si la durabilité place bien souvent le milieu physique comme un support du développement humain, la ville, en concentrant le développement de la société, se repose totalement -et parfois lourdement- sur son environnement (plus ou moins proche) pour satisfaire ses besoins : alimentation, eau, énergie, sol, matières premières et également transformées... La durabilité du milieu urbain apparait alors comme un concept purement théorique, voire une utopie technicienne (Villalba, 2009). Pour autant, l'utopie permet la définition d'un idéal certes inatteignable mais dont on peut toutefois chercher à se rapprocher. La ville durable serait alors un référentiel prospectif (Emelianoff, 2007), par rapport auquel les villes cherchent à se situer et qui peut évoluer dans le temps au cours des transactions sociales entre les acteurs et autour des projets (Hamman, 2011). Cette valeur subjective négociée entre les acteurs et autour des projets constitue alors l'objectif, normatif et moral, à atteindre. Il serait défini par une batterie d'indicateurs sur la qualité de vie, la qualité de l'environnement, la compétitivité économique, la justice sociale, l'attractivité des territoires, les externalités, ... Or pour s'approcher de cet objectif utopique projeté dans le temps long, il faut donner à la ville les moyens de gérer les multiples perturbations, qui résultent: d'interactions entre des usages parfois incompatibles; de fluctuations des ressources nécessaires à son fonctionnement ; ou de l'environnement qui l'entoure.

\subsection{La résilience urbaine}

5 À l'instar de la notion de durabilité, la résilience est un concept théorique qui a été largement discuté et modifié depuis la définition première issue de la physique des matériaux. On définit souvent cette notion comme un "umbrella concept » (Klein et al., 2004) ou encore un «boundary object» (Brand et Jax, 2007) afin de mettre en exergue sa transdisciplinarité et les approches holistiques dont elle fait l'objet lorsqu'il s'agit de la mettre en application. Parce que la recherche sur le milieu urbain regroupe des compétences diverses relevant du champ de l'urbanisme, de l'architecture, de l'ingénierie, de l'économie, de la géographie, de la sociologie..., le concept de résilience urbaine donne lieu à de multiples traductions en termes de problématique et de développement méthodologique permettant alors le dialogue (la confrontation) entre ces disciplines bien souvent segmentées. De plus, dans un contexte de forte incertitude, la capacité de la science à fournir des entrants concrets et scientifiques aux décideurs pour qu'ils mettent en place leur politique n'est plus assurée. Les échanges permanents et la co-construction du savoir et des décisions sont le fondement de la science post-normale qui devrait donc bénéficier d'un concept à la fois transversal et porteur de créativité (Strunz, 2012). Toutefois si la notion de résilience favorise les échanges et l'innovation, elle peut aussi favoriser le flou et le manque de rigueur qui nuiraient alors à son utilisation. En effet, elle est parfois définie comme un état et un processus qui y mène, ou bien comme une propriété (innée ou acquise) qui relève de diverses caractéristiques parfois contradictoires (Djament-Tran et al., 2011) : redondance, diversité, adaptabilité, interdépendance, connectivité ou encore flexibilité. Si la traduction concrète et opérationnelle de ces propriétés reste souvent difficile, «on peut, une fois qu'on les a identifiés, adopter une démarche prospective, donc opérationnelle, pour améliorer le potentiel de résilience » (Djament-Tran et al., 2011). L'ambition de la démarche engagée est donc de proposer une acception plus opérationnelle de la notion de résilience comme propriété du système urbain, qui s'appuie sur les caractéristiques précédentes et les 
traduit en indicateurs, ce qui permet ensuite de développer des outils et méthodes applicables par les acteurs de la ville pour mettre en place un processus d'amélioration de la résilience (cf. partie 3). Pour ce faire, il convient de repartir de la définition originelle de la résilience des systèmes.

6 La résilience telle que théorisée par les sciences écologiques s'appuie sur la notion de système et s'appliquerait donc à tout système : économique, écologique, humain, couplés. Elle est souvent articulée à la notion de maintien des fonctions, de persistance (Holling, 1973), rejoignant ainsi la notion de durabilité. Toutefois, la divergence des deux concepts se fait lorsque cette persistance est réalisée sans considération des systèmes extérieurs. Alors que l'objectif ultime de tout système est de se maintenir dans le temps, on ne peut pas dire qu'un système individualisé (un être humain, un animal) est durable. L'animal cherche avant tout à maximiser ses chances pour assurer sa survie, son maintien - il tend donc à être résilient - bien souvent au détriment des autres espèces. L'écosystème qui l'abrite, lui, peut être durable en mettant en place des mécanismes d'auto-régulation (qui pourrait d'ailleurs aller à l'encontre de la résilience individualisée). L'échelle spatiale de la durabilité est donc bien plus large que celle de la résilience. Cette dernière serait alors une propriété, innée et/ou acquise de tout système, une grandeur descriptive. À l'échelle d'un système micro ou mésoscopique, elle s'appréhende sur le temps court, c'est la résilience spécifique (d'un système donné face à une perturbation donnée), mais à l'échelle macroscopique, le temps long est également primordial, on parle de résilience globale (Walker et al., 2009). Or pour appliquer ce concept à la ville, il faudrait d'abord démontrer que la ville est un système puis trouver des traductions opérationnelles des notions évoquées plus haut. Si de nombreux auteurs ont effectivement tenté de modéliser la ville comme un système (de Rosnay, 2004), il n'est pas possible de représenter l'ensemble du fonctionnement urbain et de ses interactions (internes et externes) qui constituent un système complexe. Afin de dépasser ces difficultés conceptuelles et tirer profit des avantages du concept de résilience évoqués plus haut, il convient maintenant d'en proposer une définition plus opérationnelle.

7 La résilience urbaine est dans cette perspective considérée comme la capacité de la ville à absorber une perturbation puis à récupérer ses fonctions à la suite de celle-ci (Lhomme et $\mathrm{al},$. 2010). Dans cette acception, la ville est bien considérée comme un système au sens où des composants (habitats, activités, infrastructures, populations, gouvernance) interagissent pour constituer le fait urbain, mais on ne cherchera pas à décrire plus avant le système urbain. L'apparence réductrice de la définition par rapport aux définitions premières est assumée pour permettre de dépasser les difficultés conceptuelles liées à ces définitions et pour permettre aux acteurs de la ville de se saisir de la notion (on verra plus loin qu'elle permet déjà d'aborder de nombreux aspects du développement des territoires). Cette définition prend appui sur le constat que les services (ou les fonctions) à assurer par le milieu urbain font face à de nombreuses perturbations et doivent par conséquent s'adapter pour répondre à ces dysfonctionnements, c'est ce qu'on appellera la résilience de temps court. Deux leviers permettent alors d'améliorer cette résilience urbaine de temps court: 1) Une stratégie technique visant à limiter le degré de perturbation du système par une meilleure capacité de résistance et d'absorption (Serre, $2011)$; 2) Une stratégie plus organisationnelle visant à accélérer le retour à la normale par une gestion optimisée des moyens et des ressources, et une bonne accessibilité. 
Figure 1. la résilience de court terme ou la résilience spécifique d'un service urbain à une perturbation

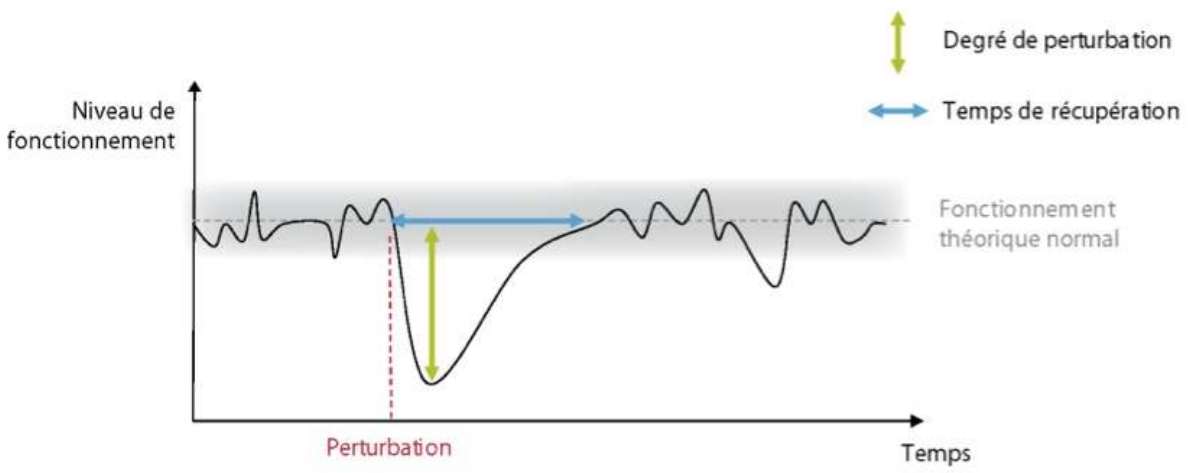

La capacité du système à fonctionner en mode dégradé (la fonction est assurée en partie ou à un niveau de performance plus faible) est un troisième levier d'action représenté par la forme de la courbe : formant alors aves les deux autres levier le triangle de la résilience (Tierney et Bruneau, 2007). Le lien avec la résilience de temps long passe logiquement par un processus d'amélioration continu qui vise à augmenter la résilience de temps court en mettant à profit les capacités d'apprentissage et d'adaptation du système pour agir sur l'un des trois leviers évoqués précédemment. Développer des méthodes d'évaluation puis d'amélioration de la résilience des systèmes urbains dans le but in fine d'opérationnaliser la notion s'inscrit dans cette perspective.

9 Par conséquent, à l'échelle du sous-système urbain (service, activité, population, ...) la résilience de temps court correspondrait à la capacité de réaction face à une perturbation. En revanche, à l'échelle globale de la ville, la résilience de temps long se fonderait sur le maintien de ses fonctions principales (prospérité, qualité de vie, attractivité, ...). Alors le concept favorise la prise en compte des impacts à long terme des décisions et des orientations données à la ville dans l'optique de mieux la préparer à maintenir ses fonctions face à une perturbation. Il ne s'agit plus de répondre à des enjeux de protection et de développement court-termistes mais d'analyser et promouvoir les mécanismes qui feront du système urbain un système apte à répondre positivement à des sollicitations futures, éventuellement inconnues.

\subsection{La ville durable doit être résiliente}

10 Ces deux concepts à la fois proches et profondément différents ont fait l'objet de nombreuses discussions concernant leur articulation. Pour certains, la résilience est une condition nécessaire à la durabilité (Folke et al., 2002 ; Klein et al., 2004). Pour d'autres, après étude des trajectoires possibles d'écosystèmes selon des états initiaux différents, la résilience n'est pas suffisante, elle n'est même pas nécessaire parfois (Derissen et al., 2011). D'après les discussions précédentes, la résilience peut jouer un rôle dans la concrétisation de la ville durable, système urbain au fonctionnement idéal. La démarche d'amélioration de la résilience urbaine est alors définie comme un moyen de gérer les soubresauts du système urbain soumis à de nombreuses perturbations (résilience de temps court) et de le maintenir dans la trajectoire idéale de la durabilité (résilience de temps long) liée à un indicateur d'état du système (la croissance économique, le bilan carbone, ou la démographie par exemple). La résilience se présente ainsi comme un 
moyen de réaliser la durabilité. Cette articulation découle logiquement des points de vue qui ont été choisis pour définir la durabilité (comme un idéal utopique) et la résilience (comme une propriété des systèmes). Il est clair que d'autres prismes d'analyses plus tournés vers les sciences sociales auraient pu mener à des acceptations différentes voire même contradictoires.

Figure 2. la résilience de long terme ou la résilience globale du système urbain : le maintien le long d'une trajectoire idéale

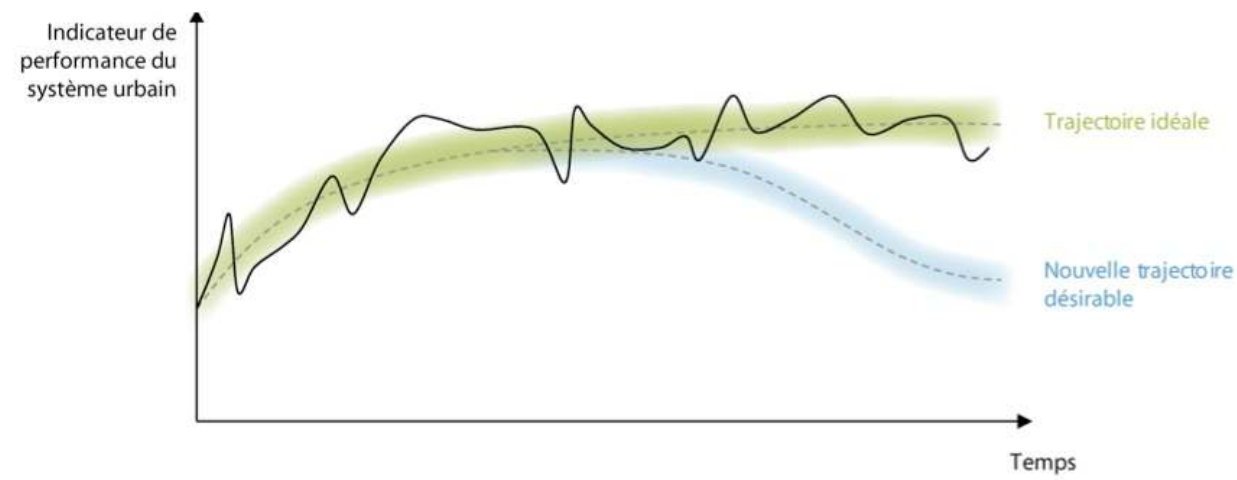

11 La possibilité de changer de trajectoire idéale doit aussi être étudiée compte-tenu des changements socio-économiques qui influent sur la stabilité du système urbain. A la faveur d'un soubresaut de prime abord perturbateur, il est possible d'utiliser les mécanismes de résilience pour ramener le système dans un état légèrement différent de l'état initial, qui initierait alors la nouvelle trajectoire. Ainsi, le mouvement des villes en transition («transition towns » parmi lesquelles Totnes, en Angleterre, fut la pionnière) se fonde sur une perturbation déjà avérée mais qui s'aggrave : la pénurie de ressources, en particulier énergétiques. Ce mouvement prône un nouvel idéal urbain défini par de nouveaux objectifs à atteindre, en particulier une empreinte écologique réduite. De même, le changement climatique, notamment à travers l'aggravation des évènements climatiques extrêmes, induit des modifications dans les possibles trajectoires du système urbain et nécessite une réorientation, i.e. une adaptation des villes.

La résilience comme capacité d'un système à s'adapter aux perturbations apparaît ainsi mieux à même de satisfaire le besoin d'opérationnaliser la ville durable. En effet, le fondement normatif du développement durable, notamment dans l'expression des grands principes planétaires, "fige " le modèle idéal à atteindre, tandis que son caractère subjectif soulève de nombreux débats quant aux valeurs - morales - à poursuivre. A l'inverse, la résilience cherche à s'affranchir des normes pour leur préférer des grandeurs descriptives et assurer une meilleure réactivité du système urbain face à l'imprévu. "Améliorer la résilience augmente les chances d'un développement durable dans un environnement changeant où le futur est imprévisible et la surprise est probable." ${ }^{2}$ (Folke et al., 2002). Pour résumer, les différences entre résilience et développement durable sont mises en regard dans le tableau 1.

Tableau 1. Caractéristiques des 2 notions

\begin{tabular}{|l|l|}
\hline RESILIENCE & DURABILITE \\
\hline
\end{tabular}




\begin{tabular}{|l|l|}
\hline Universel & Anthropocentré \\
\hline Objectif et Descriptif & Subjectif et Normatif \\
\hline De temps long et de temps court & De temps long \\
\hline Un moyen & La finalité \\
\hline
\end{tabular}

13 Finalement, comme l'a démontré (Strunz, 2012), la durabilité constitue le concept normatif cible et la résilience un concept descriptif permettant de comprendre le processus d'évolution du système qui pourra mener ou non à sa durabilité. Ainsi, la résilience peut être conçue comme une démarche opérationnelle qui permet de répondre à certains enjeux du développement urbain durable, et notamment la gestion intégrée (cf. partie 3) grâce à l'approche systémique (Voiron-Canicio, 2005).

\section{La résilience pour concrétiser la ville durable?}

La résilience définie comme la capacité à absorber puis se remettre de perturbations, a pour objectif, dans l'acception qui est la nôtre, de permettre le maintien ou l'adaptation de la trajectoire d'un système urbain dont les composantes et le fonctionnement peuvent être définis selon les principes du développement durable. Ces perturbations ont un double rôle dans la poursuite du développement urbain durable. Pour certains, la catastrophe avérée peut créer des opportunités pour une reconstruction durable (Rose, 2011). Cependant, sans attendre le désastre, sa prise en compte dès la conception de nouveaux quartiers ou dans le cadre d'opérations de renouvellement urbain donne aussi les outils et indicateurs pour assurer une meilleure résilience du système par l'adaptation du système urbain aux perturbations potentielles et inévitables. La résilience du système permet alors, face à ses perturbations, d'éviter les phénomènes de rupture, de changement de régime brutal, ou d'effondrement. Dans cette optique, les services urbains constituent l'angle d'attaque choisis dans nos travaux, ce qui ne doit pas écarter des approches plus sociales et psychologiques. En effet, dans l'articulation entre le réseau technique, le service urbain, le territoire et la population qui l'utilise, les organes de gouvernance qui l'organisent, on fait apparaître des dimensions techniques (réseau support), organisationnelle (facteurs humains dans la gestion d'un service urbain et dans la crise), sociales (comportement des usagers des services, capacités d'autonomie et d'adaptation) et aussi politiques (organisation du territoire, choix de développement des réseaux, obligations aux gestionnaires, ...). Cette approche des problématiques de risques par le prisme du fonctionnement des services urbains est à placer dans la continuité de travaux se focalisant sur les enjeux majeurs, comme par exemple (D'Ercole et Metzger, 2009 ; Demoraes, 2004).

\subsection{La nécessaire adaptation du système urbain}

Pour faire face aux nombreuses perturbations agissant sur le système urbain, l'approche résilience cherche à améliorer la capacité d'adaptation du système afin de limiter les écarts à la trajectoire idéale de la durabilité. En favorisant une approche tournée vers le long terme, prenant en compte les incertitudes sur les évolutions de l'environnement 
physique, technologique, économique et social, l'amélioration de la résilience doit anticiper l'adaptation du fonctionnement du système et de ses composants. Face à une perturbation, prévue ou non, les moyens de gérer l'instabilité du système, d'en diminuer l'intensité, d'en réduire le temps d'impact sont autant de leviers à utiliser conjointement, ou séparément, pour ramener le système dans un mode dégradé acceptable puis dans les limites normales de son fonctionnement. Si ces perturbations et les fluctuations plausibles du système urbain sont prises en compte dès la conception, alors la mise en œuvre concrète de l'adaptation sera facilitée par des composants dont les modes de fonctionnement sont flexibles ou interchangeables et par des modes de gestion intégrant l'incertitude, i.e. laissant une part d'autonomie au gestionnaire. Encore faut-il veiller en parallèle au maintien d'une vision globale des enjeux de la perturbation et à la mise en place des mécanismes collaboratifs à l'échelle du système urbain. Pour ne pas retomber dans les travers des approches axées sur l'aléa, la vulnérabilité et la protection, le risque doit être pris comme une composante et non une contrainte du développement de la ville. On l'a vu, la perturbation peut créer des opportunités qu'il faut savoir mettre à profit, et pour cela le développement même de la ville doit avoir reconnu, accepté et intégré la possibilité de perturbations, éventuellement inconnues.

L'expérience a souvent montré l'importance des réseaux techniques de la ville lors d'une catastrophe, et en particulier lors d'une inondation (Felts, 2005). En effet, ces lignes de vie (lifelines en anglais) sont nécessaires au déploiement de la ville et à sa performance car elles supportent les services essentiels dont les populations, les activités et les organes de gouvernance ont besoin (Bruneau et al., 2003): eaux, énergies, déplacements, télécommunications. Si ces services sont identifiés comme d'importance vitale pour la société et ont donc l'obligation de fiabiliser leur fonctionnement (ce que les gestionnaires parviennent généralement à faire indépendamment les uns des autres) les interdépendances entre systèmes techniques apparaissent alors rapidement comme fortement critiques. En effet, des interdépendances fonctionnelles (par exemple, le réseau de transport utilise le réseau de télécommunications pour gérer le trafic) ne se traduisent pas nécessairement par des collaborations entre les multiples gestionnaires concernés (figure 3). 
Figure 3. Des réseaux dépendants et des gestionnaires isolés

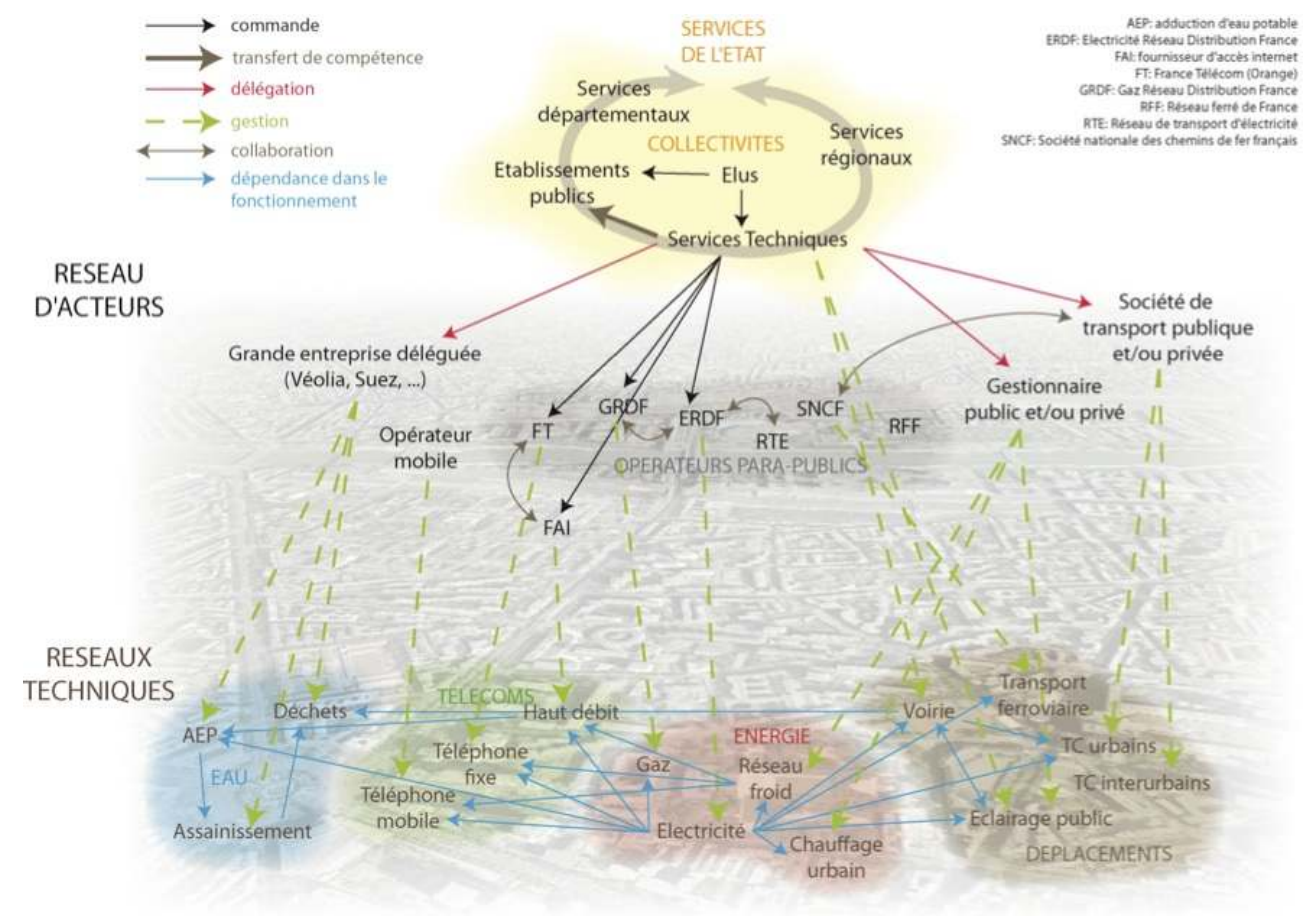

17 Cette compartimentation entre acteurs publics et privés des services de la ville est donc un frein majeur à la mise en œuvre de l'adaptation du système urbain pour en améliorer la résilience globale. Que ce soit dans l'élaboration de nouveaux aménagements ou équipements (une usine de traitement des déchets mal connectée au réseau viaire) ou lors de la gestion des situations de perturbations (l'opérateur de distribution d'énergie qui coupe l'électricité aux autres opérateurs avant qu'ils aient pu effectuer leurs mises en sécurité), le manque de collaboration entre gestionnaires remet fortement en cause la résilience du système global. Les réseaux se présentent également parmi les enjeux majeurs du développement urbain durable : transports, énergie, déchets ou eau (voir les enjeux multi-sectoriels illustrés dans les villes étudiées par (Hamman et Blanc, 2009)), d'où l'émergence d'un "génie urbain" qui ne se limite pas aux développements techniques (comme le génie civil) mais cherche à analyser de manière intégrée le rôle des réseaux, des services, des projets et des acteurs dans l'aménagement durable de la ville. C'est pourquoi l'approche systémique donnera une vision plus claire des interactions entre systèmes et permettra ensuite de prendre en compte les interdépendances entre réseaux (Lhomme et al., 2011b). De nouveaux outils et méthodes doivent donc être développés pour répondre à ces objectifs de réflexion systémique et de connaissance des interdépendances.

\subsection{Les outils et méthodes opérationnelles d'amélioration de la résilience}

Dans l'optique de déterminer les points critiques du système urbain, où des solutions d'adaptation devront être étudiées et mises en œuvre, une bonne connaissance du comportement du système urbain est requise. L'approche choisie est systémique pour mieux identifier et caractériser les interactions ayant lieu à l'intérieur du système urbain, 
mais également avec les milieux extérieurs (environnement, autres villes, ...). Dans ce système urbain, les réseaux techniques occupent une place particulière (Lhomme et al., 2010). En effet, les réseaux techniques permettent les relations entre les différents composants du système: ils sont le support des flux de personnes, d'énergies, d'informations... De manière plus complexe, et peut être moins tangible, les réseaux orientent en partie ces flux. Par exemple, en matière d'aménagement urbain, d'un point de vue purement fonctionnel, les questions d'accessibilité sont centrales et les réseaux de transport apparaissent comme structurant le territoire.

La mise en avant de la fonctionnalité du système urbain passe ainsi par la mise en exergue de l'importance des réseaux techniques pour ce fonctionnement. Or, l'étude des réseaux techniques se révèle problématique. En effet, leur fonctionnement est complexe, car les interdépendances entre réseaux techniques sont nombreuses, variées et bouclées (Rinaldi et al., 2001). C'est pourquoi, ils ne réagissent pas de façon linéaire à une perturbation. L'impact de la perturbation d'un composant peut ainsi aboutir à une chaine d'événements d'une ampleur importante, même si ce composant ne semble pas a priori majeur (Tolone, 2009). Des méthodes issues de la sûreté de fonctionnement reposant notamment sur une analyse fonctionnelle de ces réseaux, couplée à l'analyse de leur structure, de leur configuration et de leur localisation sont actuellement développées, afin d'étudier ces réseaux et leurs interdépendances (Lhomme et al., 2011a). Le croisement de ces méthodes a abouti au développement d'une méthodologie générale implémentée dans un premier prototype informatique. Plus précisément, ce prototype, permettant d'étudier la résilience des réseaux techniques, est un web SIG. Les technologies de type SIG permettent la hiérarchisation et la spatialisation d'informations de nature géographique (réseaux, zones d'aléa, bâtiments...). Cet outil permet d'analyser les impacts d'un aléa sur les réseaux techniques d'une ville, puis d'étudier la remise en service de ces réseaux à partir d'une analyse spatiale. Par exemple, des cartes (voir Figure) peuvent être produites à l'aide du prototype SIG représentant les dysfonctionnements (en rose) subits par différents réseaux (haut: eau potable; milieu: électricité; bas : assainissement) en fonction d'un scénario (les endommagements en violet). Une attention particulière est portée aux interrelations (en tireté orange) et aux dysfonctionnements occasionnés par ces interrelations (en orange).

Figure 4. Carte de réseaux théoriques représentant les interrelations entre les différents réseaux

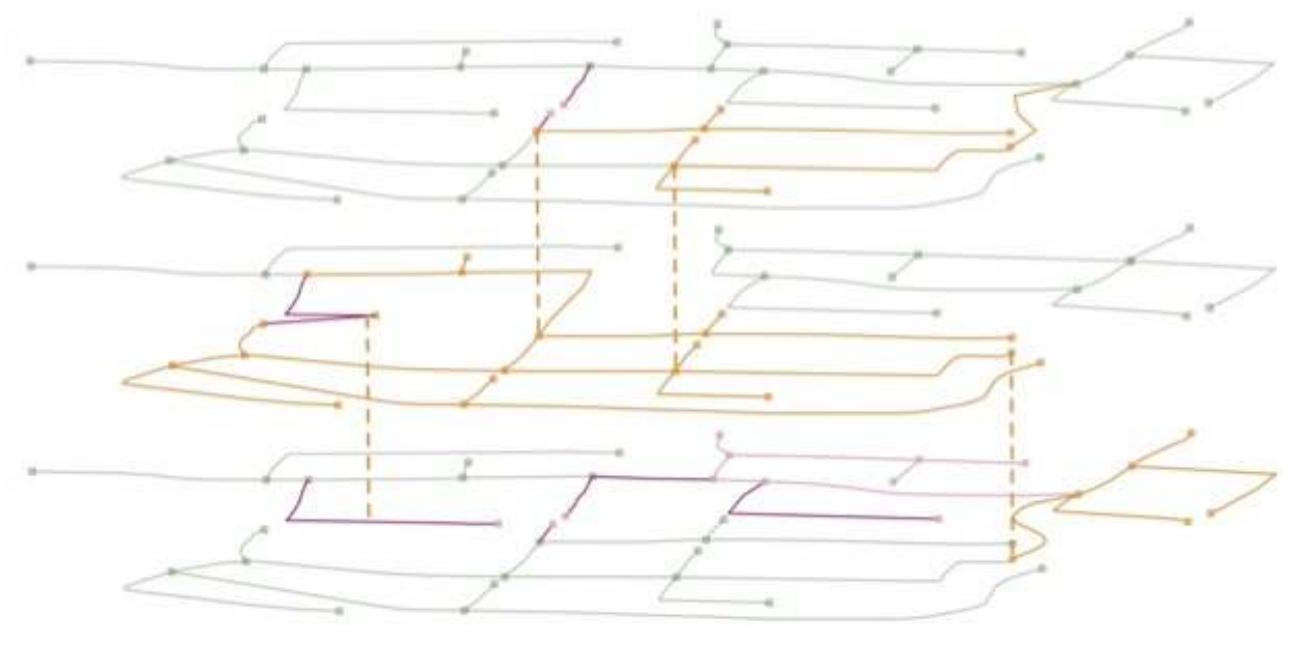


réseaux techniques ne se limitent pas au support physique qui assure le transit du flux, il s'accompagne également d'une organisation avec sa structure, ses objectifs et ses limites qui constituent le service urbain (les réseaux sont alors vus comme des « dispositifs sociotechniques de maîtrise de l'environnement (ressources, milieux) afin de satisfaire les besoins et les exigences des activités humaines. » (Le Bris et Coutard, 2009)). Ainsi l'approche technico-fonctionnelle permettant d'analyser les interdépendances est couplée avec une approche organisationnelle au niveau des acteurs. Il s'agit d'une part d'aider le gestionnaire à identifier les dépendances de son système grâce à un autodiagnostic des ressources nécessaires au fonctionnement son système et d'autre part de le sensibiliser à l'intérêt de partager ces informations avec les autres gestionnaires. En effet, la mise en commun des diagnostics est suivie d'un travail collaboratif fondé sur la représentation des dépendances entre systèmes (figure). Il permet aux gestionnaires d'échanger sur ces dépendances, d'apprendre mutuellement l'importance des ressources utilisées pour ensuite mettre en œuvre des solutions communes, tant sur le fonctionnement interne que les procédés d'échanges, la mutualisation ou la réallocation des ressources. L'intérêt de cette méthode est la construction de l'information par le gestionnaire même, qui est donc dépositaire de son exactitude et qui sera donc a priori mieux acceptée par l'ensemble des acteurs de la ville.

Figure 5. interdépendances entre 4 systèmes urbains

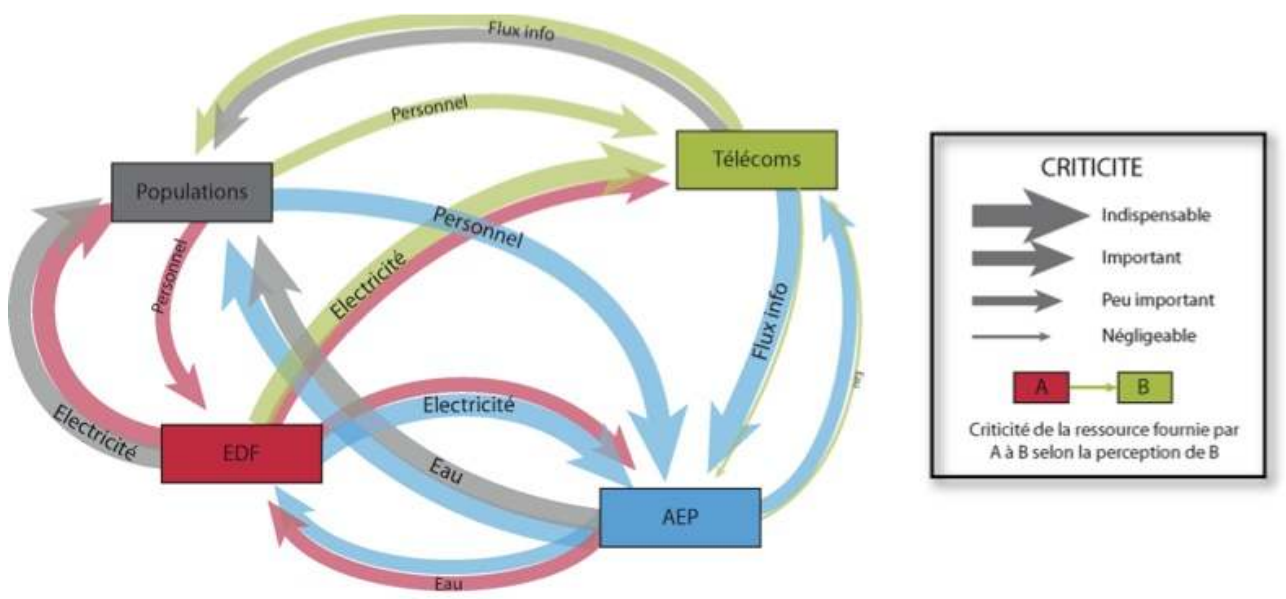

Source : Lhomme et al., 2011b

21 Pour mettre en œuvre les méthodes d'approfondissement de la connaissance du système urbain et de ses défaillances, il faut les accompagner d'outils de suivi et de gestion. Une fois les interdépendances identifiées, il s'agit de mettre en œuvre des solutions d'amélioration de la résilience des réseaux dans leur globalité ou de planifier l'aménagement du territoire et la gestion de crise de manière à tenir compte de leur comportement face à la perturbation. La gestion des interdépendances doit rester à l'échelle globale afin d'assurer la résilience de l'ensemble du système, et pas uniquement d'un sous-système. C'est pourquoi les outils développés sont dédiés aux collectivités qui devraient pouvoir donner les objectifs, coordonner les actions et les moyens pour assurer l'efficacité de la démarche. Dans la même optique que les Agenda 21, l'amélioration de la résilience urbaine pourrait faire l'objet d'une approche intégrée appuyée sur des outils de diagnostic, de suivi et d'évaluation. Le projet Resilis ${ }^{3}$ vise précisément à développer des

Développement durable et territoires, Vol. 3, n 1 | Mai 2012 
outils à destination des collectivités pour les accompagner dans la démarche d'amélioration de leur résilience. Dans la volonté d'opérationnaliser le concept, tout en l'intégrant à l'objectif final de développement urbain durable, de nombreuses limites et difficultés ont ainsi été identifiées et doivent faire l'objet de précautions particulières dans la mise en œuvre de la résilience urbaine.

\subsection{Les limites du concept}

L'intérêt du concept de résilience, défendu ici, peut toutefois être questionné. Poussée à son extrême, la résilience serait un mode opérationnel qui chercherait à limiter le coût de la protection en faisant porter les efforts sur l'adaptation et la capacité de récupération. Dès lors, l'enjeu ne serait pas de prévoir tous les risques, ni d'engager des mesures proactives de protection mais de se remettre rapidement d'une catastrophe, quels que soient les dommages subis. En allant encore plus loin, on peut voir derrière cette notion la légitimation du désengagement des institutions et la promotion de la précarité (suivant cette définition le bidonville serait la forme urbaine la plus résiliente (Djament-Tran et al., 2011), mais pas la plus durable, d'où l'intérêt du couplage des deux notions). Bien évidemment, plus les dommages sont élevés (s'il n'y a pas de mesures de protection), plus la récupération est difficile. Il en va ainsi de nos sociétés occidentales, pour lesquelles il apparait peu concevable de préconiser une telle approche. C'est la raison pour laquelle, dans le passé, des considérations coûts-bénéfices ont incité les aménageurs à tenter d'atténuer certains risques, et notamment le risque d'inondation, à des niveaux faibles, en luttant contre l'aléa. Toutefois, au-delà d'un certain seuil d'aléa, le risque ne peut plus être évité à un coût acceptable et la catastrophe est inévitable. La question de l'articulation entre le seuil d'acceptabilité et le niveau de protection est donc complexe, mais elle est écartée par l'approche portée par la résilience. Cette démarche vise en effet à stopper l'escalade des niveaux de protection sans pour autant nier l'intérêt des démarches de protection et à améliorer la capacité d'absorption pour limiter les dommages et améliorer l'acceptabilité du risque. De plus, en se limitant dans un premier temps à l'étude d'objets techniques ou socio-techniques, les critiques éthiques et morales qui pourraient être élevées (à juste titre) contre les notions de résilience sociale sont écartées.

Les discours liés à la résilience comportent une dimension performative, comme la gestion des risques avant elle (Rebotier, 2012) et comme les injonctions au développement durable maintenant. Ainsi on voit apparaître une construction du territoire urbain modelé à la fois par la gestion des risques et les objectifs de développement durable. Les acteurs de la ville se saisissent de ces enjeux pour légitimer des projets de développement, ou de non développement, qui présentent bien souvent de fortes lacunes sur l'un ou l'autre des tableaux. C'est ainsi qu'on a vu fleurir dernièrement d'innombrables éco-quartiers en zone inondable. On peut alors s'interroger sur la portée opérationnelle de ces affichages et surtout sur la contribution réelle à la durabilité et à la résilience de ces territoires. On peut justifier l'exposition d'un quartier en zone inondable d'un point de vue économique (terrains peu chers), voire environnemental, mais alors on ne peut pas s'affranchir de mesures techniques (construction de réseaux et de bâti résilients) et sociales (sensibilisation, formation et préparation des habitants au risque) accompagnant l'urbanisation. L'utilisation de ces concepts pour influer sur le territoire urbain et la société doit donc être analysée de manière critique et toujours de manière 
globale pour réellement identifier la contribution de tels projets à la résilience et à la durabilité de la ville.

Par ailleurs, certains voient à travers la notion de résilience l'idée de refuser toute évolution du système urbain en affirmant la volonté de retourner («bounce back ») à un état stable préexistant à une crise. À court terme, il paraît évident que la résilience du système urbain est le rétablissement de ses fonctions, dans un niveau de service proche de l'état antérieur. Par exemple, suite à un incident sur une usine de production d'eau potable, il n'est pas acceptable de ne produire que $60 \%$ de l'eau potable produite auparavant. Mais à plus long terme, il n'est pas certain que le système doive à tout prix retrouver ses conditions de fonctionnement antérieures. Il pourrait tout à fait se réorganiser vers un nouvel état jugé stable et désirable (Walker et al., 2009), mais pour les systèmes complexes, ces changements de trajectoire sont parfois irréversibles et les mécanismes de basculement encore méconnus. En reprenant l'exemple de l'eau potable, suite à une défaillance, il pourrait être décidé de n'utiliser l'eau potable que pour la boisson et repenser complètement notre système de production en conséquence. Par ailleurs, au lieu d'opérer un changement brutal, décision pourrait être prise d'agir sur la consommation des ménages et des industries pour diminuer la consommation d'eau potable de $20 \%$ sur 10 ans. Alors ce n'est pas la résilience qui est remise en cause puisqu'elle est le mécanisme permettant ce réajustement. C'est bien l'objectif, la trajectoire poursuivie, qui change brutalement de direction et secoue le système urbain. Dès lors, la résilience ne doit pas être pensée comme un concept carcan qui maintient le système dans des bornes figées mais plutôt comme l'outil (ressort) de retour à un état stable et désirable («bounce forward» (Manyena et al., 2011)) qui évolue dans le temps en fonction des normes sociales, environnementales et techniques. Si le cours de l'évolution veut qu'à un instant « $\mathrm{t}$ » soit définie une nouvelle trajectoire à atteindre, alors le concept de résilience indique simplement qu'il sera plus aisé et plus sain d'opérer cette bifurcation de manière progressive pour éviter la rupture et permettre au système de conserver une certaine identité.

\section{Conclusion}

Après une revue bibliographique concernant les concepts de durabilité et de résilience, il apparait que la définition choisie de la résilience urbaine permet une opérationnalisation de la durabilité urbaine. Par le maintien des fonctions urbaines dans un niveau de fonctionnement acceptable, les capacités résilientes des systèmes urbains contribuent à concrétiser les aspirations économiques, sociales et environnementales de la ville durable. Plus précisément, l'amélioration de la résilience pourrait être le moyen de rétablir l'équilibre entre les trois piliers du développement durable lorsque des perturbations remettent en question le fonctionnement social, économique ou environnemental du système urbain qui doit alors s'adapter. Qu'elle soit de temps court ou de temps long, la résilience combine les enjeux à des échelles spatiales et temporelles différentes par une vision systémique et articule les compétences de tous les acteurs de la ville. Les réseaux techniques constituent une base fondamentale pour le maintien des fonctions urbaines et font donc l'objet de développements méthodologiques opérationnels d'évaluation et de gestion à destination des collectivités locales.

Toutefois, l'implication entre la résilience et la durabilité n'est pas évidente et nécessite de bien distinguer les deux notions, et surtout leur fondement normatif ou descriptif. En 
effet, si les propriétés de résilience peuvent être recherchées, il faut bien identifier l'état vers lequel la stabilité doit être recherchée, ici la ville durable. Car comme le montrent des formes urbaines telles que les bidonvilles, la résilience n'assure pas nécessairement la durabilité globale du système. Il en va de même pour la ville qui, pour être résiliente, risque de remettre en cause la durabilité du territoire.

Des questions restent encore à résoudre, mais le concept semble un bon outil pour les expliciter et proposer des pistes de réflexion. En adoptant une définition simple de la résilience, on propose une approche concrète de l'amélioration de la durabilité urbaine. Elle se fonde sur des indicateurs et des méthodes collaboratives répondant aux enjeux de spécificité locale, de réflexion globale, intégrée et transdisciplinaire, d'adhésion des acteurs mais aussi des populations. La bonne manipulation des concepts et la bonne articulation des outils devraient permettre de concrétiser la ville durable par la ville résiliente.

\section{BIBLIOGRAPHIE}

Adger, W.N., 2003, "Building resilience to promote sustainability", Newsletter of the International Human Dimensions Programme on Global Environmental Change, $\mathrm{n}^{\circ} 2$, p. 1-3.

Brand, F.S. et Jax, K., 2007, "Focusing on the meaning(s) of resilience : resilience as a descriptive concept and a boundary object", Ecology and Society, vol. 12, n 1, $16 \mathrm{p}$.

Le Bris, C. et Coutard, O., 2009, « Les réseaux rattrapés par l'environnement ? Développement durable et transformations de l'organisation des services urbains. », Flux, vol. 74, n 4, p. 6-8.

Bruneau, M., Chang, S. E., Eguchi, R. T., Lee, G. C., O’Rourke, T. D., Reinhorn, A. M., Shinozuka, M., Tierney, K. T., Wallace, W. A. et Von Winterfeldt, D., 2003, “A framework to quantitatively assess and enhance the seismic resilience of communities", Earthquake Spectra, vol. 19, n 4, p. 733-752.

Casteigts, M., 2008, « La gouvernance des risques dans les politiques locales de développement durable : le cas des Plans communaux de sauvegarde » in : Hamman, P. (dir.), Penser le développement durable urbain : regards croisés, Paris, France, L'Harmattan, Logiques Sociales, p. 161-189.

Catnat.net, 2011, «Catastrophes naturelles : l'année 2011 sera une des plus coûteuses de l'histoire ", Données et statistiques Catnat, http://www.catnat.net/donneesstats/articles/12892catastrophes-naturelles-lannee-2011-sera-une-des-plus-couteuses-de-lhistoire.html, consulté le 09/09/2011.

Demoraes, F., 2004, Mobilité, enjeux et risques dans le District Métropolitain de Quito (Equateur), Thèse de doctorat, Géographie, Université de Savoie, Chambéry, 587 p.

Derissen, S., Quaas, M.F. et Baumgärtner, S., 2011, “The relationship between resilience and sustainability of ecological-economic systems", Ecological Economics, vol. 70, n 6, p. 1121-1128. Djament-Tran, G., Le Blanc, A., Lhomme, S., Rufat, S. et Reghezza-Zitt, M., 2011, « Ce que la résilience n'est pas, ce qu'on veut lui faire dire », http://hal.archives-ouvertes.fr/index.php? 
halsid=76e05bhugp13m9cf0o0968d0e4\&view_this_doc=hal-00679293\&version=1, consulté le 15/03/2012, $31 \mathrm{p}$.

D’Ercole, R. et Metzger, P., 2009, « La vulnérabilité territoriale : une nouvelle approche des risques en milieu urbain », Cybergéo : European Journal of Geography, Dossiers V, article 447, 16 p.

Emelianoff, C., 2007, « La ville durable : l'hypothèse d'un tournant urbanistique en Europe », L'Information géographique, vol. 71, $\mathrm{n}^{\circ}$ 3, p. 48-65.

Faytre, L., 2010, «Zones inondables : des enjeux toujours plus importants en Ile-de-France IAU », Note Rapide $n^{\circ} 518,6$ p.

Felts, L., 2005, Vulnérabilité des réseaux urbains et gestion de crise, Lyon, France, CERTU, Rapports d'étude du Certu, 78 p.

Folke, C., Carpenter, S., Elmqvist, T., Gunderson, L., Holling, C. S., Walker, B., Bengtsson, J., Berkes, F., Colding, J. et al., 2002, "Resilience and sustainable development : building adaptive capacity in a world of transformations", World summit on sustainable development, Johannesbourg, Afrique du Sud, 26 août -4 septembre 2002, 34 p.

Hamman, P., 2011, « La « ville durable » comme produit transactionnel », Espaces et sociétés, vol. $147, \mathrm{n}^{\circ} 4,25 \mathrm{p}$.

Hamman, P. et Blanc, C., 2009, Sociologie du développement durable urbain, projets et stratégies métropolitaines françaises, Bruxelles, Die Deutsche Bibliothek, EcoPolis.

Holling, C.S., 1973, "Resilience and stability of ecological systems", Annual Review of ecology and systematics, vol. 4, $23 \mathrm{p}$.

Klein, R.J.T., Nicholls, R.J. et Frank, T., 2004, "Resilience to natural hazards: how useful is this concept?", Environmental Hazards, vol. 5, p. 35-45.

Laganier, R., Villalba, B. et Zuindeau, B., 2002, « Le développement durable face au territoire : éléments pour une recherche pluridisciplinaire », Développement durable et territoire, Dossier 1 , $16 \mathrm{p}$.

Lhomme, S., Serre, D., Diab, Y. et Laganier, R., 2010, « Les réseaux techniques face aux inondations ou comment définir des indicateurs de performance de ces réseaux pour évaluer la résilience urbaine ", Bulletin de l'association des géographes français, p. 487-502.

Lhomme, S., Serre, D., Diab, Y. et Laganier, R., 2011a, “A methodology to produce interdependent networks disturbance scenarios", International Conference on Vulnerability and Risk Analysis and Management, Hyattsville, MD, USA, $10 \mathrm{p}$.

Lhomme, S., Toubin, M., Serre, D., Diab, Y. et Laganier, R., 2011b, « From technical resilience toward urban services resilience", Fourth Resilience Engineering Symposium, Nice, France, 8-10 juin 2011, p. 172-178.

Manyena, S. B., O'Brien, G., O'Keefe, P. et Rose, J., 2011, “Disaster resilience: a bounce back or bounce forward ability?", Local Environment, vol. 16, n 5, p. 417-424.

Mori, K. et Christodoulou, A., 2011, "Review of sustainability indices and indicators: Towards a new City Sustainability Index (CSI)", Environmental Impact Assessment Review, vol. 32, n 1, p. 94-106.

Rebotier, J., 2012, "Vulnerability conditions and risk representations in Latin-America: Framing the territorializing urban risk", Global Environmental Change, vol. 22, n² 2, p. 391-398.

Rinaldi, S.M., Peerenboom, J.P. et Kelly, T.K., 2001, “Identifying, understanding and analizing critical infrastructure interdependencies”, IEEE Control Systems Magazine, vol. 21, n 6, p. 11-25. 
Rose, A., 2011, "Resilience and sustainability in the face of disasters", Environmental Innovation and Societal Transitions, vol. 1, $\mathrm{n}^{\circ} 1$, p. 96-100.

de Rosnay, J., 2004, « Systémique, complexité et transdisciplinarités : nouvelles méthodes, nouveaux outils », Compte-rendu de la table ronde du 30 novembre, Futuribles International, Paris, France, 30 novembre 2004, p. 4.

Scarwell, H.J. et Laganier, R., 2004, Risque d'inondation et aménagement durable du territoire, Lille, France, Presses Universitaires du Septentrion.

Serre, D., 2011, La ville résiliente aux inondations, méthodes et outils d'évaluation, Mémoire d'HDR, Université Paris-Est, Marne-la-Vallée, 173 p.

Strunz, S., 2012, "Is conceptual vagueness an asset? Arguments from philosophy of science applied to the concept of resilience", Ecological Economics, vol. 76, p. 112-118.

Thouret, J.-C. et Leone, F., 2003, « Aléas, vulnérabilités et gestion des risques naturels » in : Morinaux V. (dir.), Les risques, Editions du Temps, p. 37-70.

Tierney, K.T. et Bruneau, M., 2007, “Conceptualizing and measuring resilience - a key to disaster loss reduction”, TR News, vol. 250, p. 14-17.

Tolone, W.J., 2009, “Interactive visualizations for critical infrastructure analysis”, International Journal of Critical Infrastructure Protection, vol. 2, n 3, p. 124-134.

Villalba, B., 2009, "L'utopie sociale de la ville durable », EcoRev' - Revue critique d'écologie politique, consulté le 11 juin 2012. URL : http://ecorev.org/spip.php?article648

Voiron-Canicio, C., 2005, « Pour une approche systémique du développement durable », Développement durable et territoires, Dossier $4:$ La ville et l'enjeu du Développement Durable, mis en ligne le 30 mai 2005, consulté le 11 juin 2012. URL : http://

developpementdurable.revues.org/261

Walker, B., Abel, N., Anderies, J. et Ryan, P., 2009, "Resilience, adaptability, and transformability in the Goulburn-Broken catchment, Australia", Ecology and Society, vol. 14, n 1, 12 p.

\section{NOTES}

1. "The unfocussed aspirations for sustainability are captured in the notion of resilience - the ability to persist and the ability to adapt."

2. "Managing for resilience enhances the likelihood of sustaining development in changing environments where the future is unpredictable and surprise is likely."

3. Resilis est un projet ANR (ANR-09-VILL-0010-VILL) mené par Egis en partenariat avec EIVP, Iosis, Sogreah, Cemagref, REEDS, LEESU, Fondaterra. (www.resilis.fr)

\section{RÉSUMÉS}

Le concept de résilience réinterroge la façon de penser le système urbain et ses perturbations. Appliqué à la ville, il peut être défini comme la capacité d'un système urbain à absorber une 
perturbation et à retrouver ses fonctions à la suite de cette perturbation. Dès lors, l'opérationnalité du concept passerait par la nécessité d'adapter le fonctionnement du système urbain tout comme ses composants aux perturbations potentielles, à reconstruire le système urbain suite à une perturbation majeure ou à définir des modalités de gestion de crise en intégrant la complexité même de la ville. Ainsi le concept de résilience appliqué à la ville semble trouver des traductions opérationnelles, notamment en matière de services urbains, qui répondent également aux objectifs de durabilité. En partant de l'analyse des définitions des deux concepts de durabilité et de résilience appliqués à la ville, l'objectif de cet article est de s'interroger sur la façon dont s'articulent les concepts de résilience et de ville durable puis de proposer des outils et méthodes contribuant à la résilience de la ville dans une optique de durabilité, avant d'en étudier les limites.

Urban resilience questions the way we think urban systems and disturbances. Applied to cities, resilience is the ability of a city to absorb a disturbance and recover its functions after the disturbance. Then, urban systems need to adapt their functioning and their components to possible disturbances. In managing the crisis and organizing the recovery process, the complexity of urban systems must also be taken into account. Then, it seems that translating the theoretical concept of resilience into practice is possible if functioning, interactions, disturbances and stakeholders are analyzed together, which should contribute to urban sustainability at the same time. Urban services are a relevant case study in doing so. We first review the definitions of both concepts: sustainability and resilience, in order to discuss the conceptual links between them. Then we propose methods and tools dedicated to practioners involved in making cities resilient and sustainable and we discuss the limits of the concepts.

\section{INDEX}

Keywords : interdependencies, sustainable city, urban resilience, urban services

Mots-clés : résilience urbaine, ville durable, services urbains, interdépendance

\section{AUTEURS}

\section{MARIE TOUBIN}

Marie Toubin, Doctorante CIFRE avec Egis, en collaboration avec l'Ecole des Ingénieurs de la Ville de Paris (Université Paris-Est Marne-la-Vallée) et l'Université Paris 7 Paris Diderot, Laboratoire PRODIG (Pôle de Recherche pour l'Organisation et la diffusion de l'Information Géographique), UMR CNRS 8586. EIVP, 15 rue Fénelon, 75010 Paris. Marie.toubin@eivp-paris.fr

\section{SERGE LHOMME}

Serge Lhomme, Doctorant en Génie Urbain à l'Ecole des Ingénieurs de la Ville de Paris en collaboration avec l'Université Paris-Est Marne-la-Vallée et l'Université Paris 7 Paris Diderot, Laboratoire PRODIG (Pôle de Recherche pour l'Organisation et la diffusion de l'Information Géographique), UMR CNRS 8586. EIVP, 15 rue Fénelon, 75010 Paris. Serge.lhomme@eivp-paris.fr

\section{YOUSSEF DIAB}

Youssef Diab, Directeur scientifique de l'Ecole des Ingénieurs de la Ville de Paris, Professeur de génie civil urbain à l'Université Paris-Est Marne-la-Vallée. EIVP, 15 rue Fénelon, 75010 Paris. Youssef.diab@univ-mlv.fr 


\section{DAMIEN SERRE}

Damien Serre, Enseignant-Chercheur HDR à l'Ecole des Ingénieurs de la Ville de Paris, responsable de l'axe de recherche « résilience urbaine ». Ses recherches ont pour finalité la formalisation des connaissances utiles pour l'aide à la décision et pour l'aide à la conception de villes résilientes face aux risques. Sa recherche est transdisciplinaire et au service de la ville. EIVP, 15 rue Fénelon, 75010 Paris. Damien.serre@eivp-paris.fr

\section{RICHARD LAGANIER}

Richard Laganier, Professeur de Géographie à l'Université Paris 7 Paris Diderot, Sorbonne Paris Cité, laboratoire PRODIG (Pôle de Recherche pour l'Organisation et la diffusion de l'Information Géographique), UMR CNRS 8586. Son activité de recherche porte sur l'étude des rapports entre risques liés à l'eau et territoire et l'analyse des conditions de développement de la résilience. Il est auteur ou co-auteur de nombreux ouvrages sur les extrêmes hydrologiques et leur gestion. Univ. Paris 7, 7 rue Watt, 75013 Paris. Richard.laganier@univ-paris-diderot.fr 